\title{
Valores conflitantes na produção do DSM-5: o "caso" da síndrome psicótica atenuada
}

\author{
Arthur Maciel Nunes Gonçalves*1 \\ Clarissa de Rosalmeida Dantas*2 \\ Cláudio E. M. Banzato*3
}

\begin{abstract}
$O$ debate sobre a possível inclusão da síndrome psicótica atenuada como diagnóstico oficial no DSM-5 foi atravessado por diversos valores em torno dos quais argumentadores a favor ou contra a criação dessa nova categoria se dividiram. As defesas de tais valores geraram posições divergentes, mas igualmente legítimas e bem fundamentadas, e trouxeram consigo questionamentos importantes para o cenário potencialmente inovador da psiquiatria preventiva contemporânea. Entre eles, a preocupação ética com o sofrimento, os riscos associados às ações de prevenção e a importância do papel dos valores na formulação de categorias diagnósticas e na própria prática psiquiátrica.
\end{abstract}

Palavras-chave: Síndrome psicótica atenuada, valores, DSM-5, estado mental de risco

${ }^{{ }^{1}, 2,3}$ Universidade Estadual de Campinas - Unicamp (Campinas, SP, Br). 


\section{Introdução}

Em 26/11/2013, Allen Frances, chefe da força-tarefa que produziu o DSM-IV, publicou no site The Huffington Post que "a estrada para o inferno é pavimentada com boas intenções e más e inesperadas consequências". Sua afirmação era uma crítica aberta à possibilidade de inclusão no corpo principal do DSM-5 da Síndrome Psicótica Atenuada (SdPA), uma nova categoria diagnóstica criada para identificação de casos em risco para a psicose (Frances, 2013). O mesmo autor, em uma publicação na revista Psychiatric Times intitulada "Abrindo a caixa de Pandora: as 19 piores sugestões para o DSM-5", declarou considerar a SdPA como a proposta "mais preocupante" dos revisores do novo manual, e, no blog da revista Psychology Today, mencionou que até 2009 estava decidido a não fazer comentários sobre a revisão da quinta edição do DSM, mas que se sentiu compelido a falar ao perceber que a nova proposta de categoria tinha chances reais de se tornar um diagnóstico psiquiátrico oficial. Para Frances (2010a, 2010b), embora a intenção de se criar a SdPA fosse "nobre" e "admirável", os membros do Grupo de Trabalho de Transtornos Psicóticos do DSM-5 não estavam dando peso suficiente às muitas consequências perigosas que poderiam advir com a oficialização desta categoria.

Em maio de 2012, por recomendação do Grupo de Trabalho, foi anunciado no site dedicado ao DSM-5 que a SdPA seria incluída na Seção III do manual, área do documento reservada para condições a serem melhor estudadas (American Psychiatry Association, 2013). Tal notícia gerou alívio em muitos comentadores e insatisfação em outros, confirmando que o campo de discussão, tanto em meios científicos quanto não científicos, estava marcado por posições conflitantes (Maxmen, 2012). Para além de discussões puramente científicas ou factuais sobre a SdPA, o debate sobre sua inclusão no DSM-5 foi atravessado por diversos valores, em torno dos quais os argumentadores a favor ou contra a criação desse novo diagnóstico se dividiram. Embora "valores" seja um termo complexo e de definição imprecisa, para nossos propósitos adotaremos a visão do filósofo de Oxford, Richard Hare, de que valores são termos relativos a quaisquer julgamentos de bom e ruim (Hare, 1996). Tais julgamentos apresentam um caráter eminentemente prescritivo, ou seja, indicam uma determinada direção para a ação, e dizem respeito a diversas áreas de atuação ou interesses humanos, de maneira a existirem diferentes tipos de 
valores: estéticos, morais, pragmáticos etc. Por entender que esses elementos estão implícitos, em maior ou menor grau, nas atividades cotidianas e que influenciam as tomadas de decisões, alguns autores têm sugerido a análise de valores como meio de apreender a dinâmica de conformação da psiquiatria, seja em suas classificações diagnósticas, na própria prática clínica e em outras áreas de atuação da especialidade (Sadler, 2005; Woodbridge-Dodd, \& Fulford, 2004). No caso do debate em torno da SdPA, a defesa de múltiplos valores gerou posições divergentes, mas igualmente legítimas e bem fundamentadas, e a falta de conciliação entre essas visões fomentou uma discussão acalorada, que trouxe consigo questões significativas para o cenário potencialmente inovador da psiquiatria preventiva contemporânea.

\section{O ônus da psicose e a formulação dos estados clínicos de risco}

Sabidamente os quadros psiquiátricos causam intenso sofrimento e se iniciam, em sua maioria, na adolescência e na vida adulta jovem. Associam-se com frequência a altas taxas de incapacidade, muitas vezes permanente, com empobrecimento do funcionamento social e familiar, prejuízos acadêmicos, instabilidade profissional e desemprego (McGorry et al., 2007). Em destaque, os quadros psicóticos, especialmente a esquizofrenia, têm sido alvo de atenção por parte de clínicos e pesquisadores há décadas, por terem impacto devastador na qualidade de vida das pessoas acometidas (McGorry, 1998). Motivados pelo intuito de minimizar o sofrimento vivido por esses pacientes, psiquiatras ao redor do mundo têm buscado melhores formas de cuidado, tentando especialmente encurtar o tempo entre o surgimento dos sintomas psicóticos e o início do tratamento. Mas, em consonância com o movimento preventivo desenvolvido pelas demais especialidades médicas e por outras profissões da área da saúde, além de procurar maneiras de diagnosticar e tratar mais rapidamente os pacientes, alguns autores vêm buscando identificar grupos de indivíduos cujos sintomas representem uma condição clínica de risco para a psicose. A ideia por trás desse movimento é a de minimizar potenciais prejuízos cognitivos, funcionais e sociais, de promover bem-estar aos pacientes ${ }^{1}$ e, se possível, até evitar a conversão para um quadro psicótico franco (Yung, \& McGorry, 1996).

Embora a preocupação com o reconhecimento de casos "pré-psicóticos" remonte a 1927, com as observações do psiquiatra e psicanalista Harry Stack

${ }^{1}$ A intenção de ampliar o bem e reduzir o mal na vida daqueles que sofrem guarda relação com o princípio ético da beneficência, segundo Beauchamp, \& Childress (2002, p. 282), e também com o valor que Sadler (2005, p. 446) nomeou como 'auxílio aos enfermos'. 
Sullivan, foi a partir da década de 1990 que um novo paradigma de pesquisa começou a ser implementado (McGorry, 1998). As investigações iniciais se debruçaram sobre uma revisão conceitual do momento de emergência dos sintomas indicativos de psicose. A princípio, esse período foi chamado de "estado prodrômico", por se entender que os sintomas presentes, mesmo que inespecíficos, já seriam constituintes de um quadro psicótico em surgimento. ${ }^{2}$ Entretanto, estudos mostraram que só uma minoria dos indivíduos com os sintomas descritos efetivamente convertia para um quadro psicótico característico, falsificando a ideia de que essas manifestações eram sempre "prodrômicas". Além disso, havia outra questão conceitual que impedia o uso do termo: por definição a identificação do período prodrômico é retrospectiva, indo na contramão do ideal de reconhecimento prospectivo dos pacientes candidatos à conversão para psicose. A partir daí foi formulado um novo conceito e o "estado prodrômico" passou a ser chamado de "estado mental de risco" (at-risk mental state), segundo o qual os pacientes identificados apresentavam sintomas que poderiam, mas não necessariamente iriam, converter-se em sintomas psicóticos propriamente ditos (Yung et al., 1996).

A importância dos estados mentais de risco foi reconhecida de tal maneira que culminou como proposta de uma nova categoria diagnóstica para o DSM-5, a Síndrome de Risco para Psicose (Fusar-Poli et al., 2013). Sugerida logo à época da fundação do Grupo de Trabalho dos Transtornos Psicóticos do novo manual, ela foi rapidamente considerada "prematura" e "confusa", pois, chamando-a de "síndrome de risco", subentendia-se que os sintomas apresentados eram somente fatores de risco, sugerindo a ideia de que, por si só, não precisavam de cuidados clínicos. Estudos, ao contrário, mostravam que os pacientes atendidos e identificados como "em risco" já necessitavam de tratamento para o sofrimento trazido pelos sintomas, ou seja, necessitavam mais do que somente intervenções preventivas. $\mathrm{O}$ nome do diagnóstico foi mudado então para Síndrome Psicótica Atenuada e a nova nomenclatura passou a refletir o quadro sintomático de uma população que já requeria tratamento. Conceitualmente deixou de representar uma síndrome de risco para assumir status de transtorno mental (Phillips, 2013).

A ideia de se oficializar a SdPA como um diagnóstico psiquiátrico que representasse, pelo menos em parte, os achados de duas décadas de pesquisa na área de identificação de estados mentais de risco ampliaria o acesso ao cuidado apropriado para os pacientes nessa condição, além de possibilitar um maior financiamento de

${ }^{2} \mathrm{O}$ pródromo de uma doença é caracterizado por manifestações clínicas que precedem a eclosão dos seus sintomas principais e mais peculiares, assim como a tosse e a coriza precedem em cerca de três a quatro dias a eclosão das manchas vermelhas na pele do paciente com sarampo (Yung et al., 1996). 
pesquisas clínicas para novas descobertas no campo. Foi em favor dessas repercussões que muitos psiquiatras, e até familiares de pacientes, aguardavam a aprovação do Grupo de Trabalho de Transtornos Psicóticos do DSM-5 para a inclusão da categoria no manual (Costello, 2012; McGlashan, \& Woods, 2011). Embora ainda necessitando de replicação de estudos e comparação de resultados, hipoteticamente as intervenções precoces poderiam minimamente retardar a eclosão de quadros psicóticos e, na melhor das situações, até evitar seu surgimento.

Diante da rejeição dessa categoria do corpo principal do manual, uma pergunta emerge: além da intenção de fazer o bem e minimizar o mal na vida dos pacientes, quais outros valores estavam em jogo nessa decisão?

\section{O risco da categoria de risco: estigma, tratamento e a definição do bem}

Foi pela dúvida sobre as possíveis consequências prejudiciais da SdPA que muitos autores se posicionaram contra sua inclusão no corpo central do DSM-5. Uma das principais preocupações em relação ao tema se dirigia às evidências de que somente 10 a $30 \%$ dos indivíduos que preenchiam os seus critérios diagnósticos progrediriam para um quadro psicótico franco, gerando rotulação estigmatizante e tratamento desnecessário a muitos jovens (Fusar-Poli et al., 2014). Para diversos críticos, especialmente Allen Frances, o investimento na oficialização da SdPA e o uso de antipsicóticos, com seus potenciais efeitos colaterais como ganho de peso, predisposição a problemas cardiovasculares e potencial redução da expectativa de vida em jovens que jamais apresentariam um quadro psicótico, promoveria alocação inapropriada ${ }^{3}$ dos já escassos recursos financeiros da saúde mental e acarretaria danos incalculáveis à sociedade (Frances, 2010a). Para ele e outros autores os impactos da estigmatização sobre o sentido de autonomia ${ }^{4}$ e responsabilidade dos jovens tratados seriam imprevisíveis, inclusive do ponto de vista forense (Oliveira, 2012).

Em clara defesa da SdPA, o chefe do Grupo de Trabalho de Transtornos Psicóticos, William Carpenter, tentou destacar alternativas aos riscos potenciais oferecidos pelos antipsicóticos, apontando a necessidade de se investir no uso de

${ }^{3}$ Do ponto de vista da ética médica, interferir inapropriadamente na alocação de recursos financeiros dos sistemas de saúde seria uma não observância ao princípio da justiça, mais especificamente da justiça distributiva (Beauchamp, \& Childress, 2002, p. 352).

${ }^{4} \mathrm{O}$ respeito pela capacidade de pessoas autônomas de tomarem suas próprias decisões corresponde, na ética médica, ao princípio da autonomia que, junto com a beneficência, a não maleficência e a justiça, compõe os quatro princípios fundamentais da bioética (Beauchamp, \& Childress, 2002, p. 137). 
medicamentos mais seguros para o tratamento dos pacientes diagnosticados. Entre eles estava o ômega-3, um ácido graxo polinsaturado encontrado naturalmente em alguns alimentos, que administrado em doses elevadas demonstrou, em uma pesquisa, ser capaz de reduzir de $28 \%$ para $5 \%$ o risco de conversão para psicose sem efeitos colaterais significativos (Amminger et al., 2010). Para Carpenter, caso esse estudo fosse replicado e houvesse confirmação do benefício do ômega-3, haveria significativamente menos preocupações em relação à geração de danos às pessoas diagnosticadas com a SdPA que não convertessem para a psicose (Maxmen, 2012). Entretanto, até a data de decisão sobre a oficialização da SdPA no manual, o estudo não havia sido replicado e a questão da estigmatização ainda continuava aberta.

Embora a inclusão da SdPA no DSM-5 tenha sido inicialmente incentivada por muitos pesquisadores experts da área como uma atitude potencialmente benéfica, alguns deles retiraram formalmente seu apoio à iniciativa, justamente por compreender que não havia um consenso sobre o que se propunha como tratamento para os casos de risco identificados. Era precisamente na indefinição de que tipos de tratamento representavam "fazer o bem" que residia o principal risco da SdPA. Esse desconforto também ficou evidente com a declaração de Patrick McGorry (2012), importante psiquiatra da infância e adolescência australiano, que apontou para a necessidade de se romper com a ideia prevalente nos EUA de que o tratamento medicamentoso era a principal ou a única forma de intervenção para os pacientes, "um nexo reforçado pelo rígido reducionismo neurobiológico que tomou conta da psiquiatria americana desde os anos de 1980” (p. 1).

\section{Oficialização, reificação e medicalização}

Discute-se na literatura psiquiátrica que quando um conceito diagnóstico é listado oficialmente em um manual classificatório e apresentado com uma definição operacional, há uma tendência de reificação ${ }^{5}$ desse construto (Hyman, 2010; Mishara, \& Schwartz, 2013). Entre os riscos da reificação das categorias psiquiátricas oficiais está o impedimento de avanços científicos, tanto pela destinação preferencial de verbas a pesquisas que utilizem essas categorias como diagnósticos de referência, quanto por transformá-las em "viseiras epistêmicas", limitando a formulação teórica, e até mesmo a imaginação, de modelos alternativos sobre o adoecimento mental.

${ }^{5}$ Reificação é entendida nesse contexto como a compreensão e utilização dos diagnósticos psiquiátricos na forma de objetos concretos, entidades com existência própria (do latim rēs, rēi, "coisa"), e não como formulações abstratas e hipotéticas sobre a realidade (Hyman, 2010; Banzato, 2015). 
Outro risco associado refere-se ao efeito exercido sobre a configuração das próprias experiências subjetivas dos clínicos e pacientes, moldando-as de acordo com o pano de fundo das categorias diagnósticas reificadas (Banzato, 2015; Hyman, 2010). Considerando o reducionismo neurobiológico presente na cultura psiquiátrica norte-americana, como mencionado por McGorry, a utilização da SdPA de maneira reificada muito provavelmente seria associada, na prática, à prescrição de medicamentos e a efeitos desconhecidos na subjetivação dos pacientes diagnosticados.

Para Frances, o Grupo de Trabalho sempre esteve muito bem intencionado em suas propostas e os especialistas que o compunham dificilmente cometeriam o erro de reificar a SdPA e de prescrever inadequadamente medicamentos para tratá-la. Mas, na opinião do psiquiatra norte-americano, não seria responsável levarem adiante sugestões de um diagnóstico que fosse utilizado de maneira segura apenas por especialistas, como eles próprios. Caso a SdPA se tornasse uma categoria oficial no DSM-5, não haveria controle sobre o marketing realizado pela indústria farmacêutica, sobre a forma de operacionalização deste conceito, nem sobre prescrição dos psiquiatras em sua prática cotidiana (Frances, 2010a).

É importante notar que grande parte das críticas dirigidas à inclusão da SdPA se relaciona aos múltiplos sentidos do que se tem chamado de medicalização (Oliveira, 2012; Silva, 2013; Zorzanelli et al., 2014). A busca por intervenções precoces em um grupo de pacientes com sintomas atenuados e indicativos de conversão para um quadro mais grave pode ser compreendida, partindo-se de uma perspectiva exterior à profissão, como a apropriação pelo discurso psiquiátrico de comportamentos mal-adaptados, associada à redefinição da população -alvo de suas intervenções, com a expansão dos interesses que partiam dos casos severos e crônicos, indo em direção aos menos graves ou até assintomáticos. Esse movimento seria expressão de uma ampliação dos limites do conceito de morbidade na medicina, chegando ao ponto de incluir o risco da doença como objeto de tratamento (Zorzanelli et al., 2014).

Embora Frances e outros críticos não demonstrassem acreditar que a criação da SdPA fosse uma expansão intencional da área de atuação psiquiátrica, na linha do controle social e de um imperialismo médico, sua preocupação era de que isso ocorresse mesmo de maneira não intencionada, como aconteceu com outros diagnósticos no passado (Oliva, 2014). Sua preocupação era agravada pela constatação de que o diagnóstico da SdPA se dirigiria fundamentalmente a grupos muito vulneráveis, como adolescentes e adultos jovens em sofrimento mental, e ainda mais na presença e mobilização ativa de outros atores fora da profissão médica, como a indústria farmacêutica e até os familiares de pacientes (Costello, 2012; Frances, 2010a). Essa visão vai ao encontro de definições mais recentes de medicalização, segundo as quais ela é compreendida como um processo irregular, complexo e que conta com múltiplos e variados agentes sociais (Zorzanelli et al., 2014). 


\section{A rejeição da categoria como priorização valorativa}

A despeito de um longo e intenso debate, que incluiu valores como a intenção de se fazer o bem, a preocupação com o potencial de se causar danos, os interesses econômicos, as conflitantes visões sobre a natureza do adoecimento mental e suas possibilidades de tratamento, a rejeição da SdPA se deu oficialmente pela falta de dados sobre a confiabilidade diagnóstica da categoria (Yung et al., 2012). O Grupo de Trabalho de Transtornos Psicóticos analisou os resultados de um pequeno estudo, no qual foi solicitado a alguns médicos que avaliassem isoladamente se os pacientes a eles apresentados seriam diagnosticados ou não com a SdPA, baseados nos critérios propostos para o DSM-5 (Regier et al., 2013). Em uma reunião da Associação Psiquiátrica Americana, Darrel Regier, vice-presidente da força-tarefa do novo manual, revelou que os resultados do estudo foram estatisticamente pouco atrativos, levando o Grupo de Trabalho a não apoiar a inclusão do diagnóstico. A avaliação havia incluído somente sete pacientes, motivo pelo qual Carpenter, chefe do grupo, diz não ter sido efetiva. Ele, que não pôde estar presente na última reunião, realizada em abril de 2012, declarou que se estivesse lá "teria tentado arduamente preencher a lacuna da confiabilidade com mais dados" (Maxmen, 2012, p. 1). Embora não fizesse objeção à decisão, não se dizia entusiasmado em relação a ela (ibid.).

Os dados desse estudo envolvendo a SdPA foram divulgados oficialmente em janeiro de 2013, junto com os resultados das análises de outras categorias, em uma publicação escrita por Regier e colaboradores. Nesse documento havia uma declaração informando que, embora o método de amostragem utilizado nas pesquisas do DSM-5 tenha sido superior aos utilizados em revisões anteriores do manual, ele não foi efetivo em obter amostras de tamanho adequado para a análise de algumas das categorias e que essa era uma "limitação significativa" do estudo (Regier et al., 2013). Curiosamente, nessa mesma publicação havia uma afirmação de que os ensaios de campo tinham como único intuito prover informações para o processo de tomada de decisão a respeito do DSM-5, e que por si só não poderiam determinar a inclusão ou exclusão de diagnósticos na versão final do manual (Clarke et al., 2013). Mesmo diante dessa informação, ficou a cargo dos estudos de campo a razão aparentemente determinante para a não oficialização da SdPA. É de se questionar por que a força-tarefa do DSM-5, a princípio defensora do rigor científico como um de seus valores mais importantes, permitiu que a investigação sobre a confiabilidade dessa categoria envolvesse somente sete pacientes, ainda mais se levarmos em conta inúmeros indivíduos com esse diagnóstico acompanhados nos centros especializados em estados mentais de risco para psicose ao redor do mundo. 


\section{OBSERVANDO A PSIQUIATRIA}

\section{Conclusão}

A análise do debate em torno da SdPA revelou uma multiplicidade de valores defendidos por atores variados, cada qual com sua legitimidade e importância. A discussão trouxe uma preocupação ética com o sofrimento humano, relembrando que aqueles que sofrem ou acompanham indivíduos em sofrimento podem apresentar um desejo legítimo de minimização da dor e, quando possível, sua evitação. Sob outro ângulo, trouxe também o alerta de que as mesmas práticas de prevenção suscitam questões sobre os riscos a elas associados e sobre os limites da ação dos profissionais de saúde e da regulação das condutas.

Os valores estão presentes em todas as atividades humanas, mas como nos lembram Woodbridge-Dodd \& Fulford (2004), é especialmente quando os valores entram em conflito que notamos sua presença. Para esses e outros pesquisadores, ter a percepção dos valores atuantes é o primeiro passo para se tomar decisões mais equilibradas, que levem em conta, e com mesmo peso, as vozes dos atores envolvidos, com suas motivações e interesses diversos, e também as evidências científicas. Os autores nos lembram que, mesmo com abordagens baseadas em princípios como a da ética médica, não há soluções pré-definidas para a resolução de conflitos valorativos, e que é preciso construir um processo adequado de tomada de decisões, em vez de uma prescrição a priori de valores e resultados "corretos".

Por fim, vale apontar que a análise de valores do debate em torno da SdPA lembra, em larga medida, pontos de intensa discussão vividos em torno de outras categorias do DSM-5 e também por toda a psiquiatria. Provavelmente tal coincidência se deva ao fato de que falar de valores na psiquiatria é falar da própria história e constituição da disciplina como prática médica. Mesmo que denominados de outras formas, os valores estiveram sempre presentes e reconhecê-los e articulá-los parece ser uma ferramenta ao mesmo tempo necessária e promissora para o avanço da profissão.

\section{Referências}

American Psychiatry Association. DSM-5 Development. Recuperado em 21 mar. 2013 de: <http:// www.dsm5.org/Pages/Default.aspx>.

Amminger, G.P. et al. (2010). Long-Chain $\omega$-3 Fatty Acids for Indicated Prevention of Psychotic Disorders - A Randomized, Placebo-Controlled Trial. Archives of General Psychiatry, 67(2), 146. doi:10.1001/archgenpsychiatry.2009.192.

Banzato, C.E.M. (2015). Reification. In R.L. Cautin, \& S.O. Lilienfeld (Eds.), The Encyclopedia of Clinical Psychology. (pp. 1-4). Malden, Oxford: John Wiley and Sons Inc. doi:10.1002/9781118625392.wbecp168. 
Beauchamp, T.L., \& Childress, J.F. (2002). Princípios de ética biomédica. São Paulo: Loyola. Clarke, D. E. et al. (2013, Jan.). DSM-5 field trials in the United States and Canada, part I: Study Design, Sampling Strategy, Implementation and Analytic Approaches. American Journal of Psychiatry, 170, 44-58.

Costello, V. (2012). Final DSM-5 Puts Attenuated Psychosis Syndrome in "Needs More Review"... Means It is Not a Reimbursable Diagnosis. Recuperado em 2 set. 2014 de: <http:// mentalhealthmomblog.com/2012/12/03/dsm-5-finalized-and-it-keeps-the-proposed-new-diagnosis-of-attenuated-psychosis-disorder/>.

Frances, A. (2010a). Psychosis Risk Syndrome: Just As Risky With A New Name. Recuperado em 26 ago. 2014 de: <http://www.psychologytoday.com/blog/dsm5-in-distress/201007/ psychosis-risk-syndrome-just-risky-new-name $>$.

Frances, A. (2010b). Opening Pandora's Box: The 19 Worst Suggestions For DSM5. Recuperado em 21 jun. 2014 de: <http://www.psychiatrictimes.com/display/article/10168/1522341>.

Frances, A. (2013). Psychosis Risk Syndrome Is Back To Haunt Us. Recuperado em 26 ago. 2013 de: <http://www.huffingtonpost.com/allen-frances/psychosis-risk-syndrome-i_b_4343661. html $>$.

Fusar-Poli, P. et al. (2013). The psychosis high-risk state: a comprehensive state-of-the-art review. JAMA psychiatry (Chicago, Ill.), 70(1), 107-120. doi:10.1001/jamapsychiatry.2013.269.

Fusar-Poli, P. et al. (2014). Attenuated psychosis syndrome: ready for DSM-5.1? Annual review of clinical psychology, 10, 155-192. doi:10.1146/annurev-clinpsy-032813-153645.

Hare, R.M. (1996). A linguagem da moral. São Paulo: Martins Fontes.

Hyman, S.E. (2010). The diagnosis of mental disorders: the problem of reification. Annual review of clinical psychology, 6, 155-179. doi:10.1146/annurev.clinpsy.3.022806.091532.

Maxmen, A. (2012). Psychosis risk syndrome excluded from DSM-5. Recuperado em 31 ago. 2014 de: <http://www.nature.com/news/psychosis-risk-syndrome-excluded-from -dsm-5-1.10610>.

McGlashan, T.H., \& Woods, S. (2011). Early Antecedents and Detection of Schizophrenia. Recuperado em 2 set. 2014 de: <http://www.psychiatrictimes.com/schizophrenia/early-antecedents-and-detection-schizophrenia $>$.

McGorry, P. (2012). APA to leave Attenuated Psychosis Syndrome out of DSM-5 Main Text. Recuperado em 2 set. 2014 de: < http://www.patmcgorry.com.au/blog/pmcgorry/apa-leave -attenuated-psychosis-syndrome-out-dsm-5-main-text $>$.

McGorry, P.D. (1998). "A stitch in time”...the scope for preventive strategies in early psychosis. European Archives of Psychiatry and Clinical Neuroscience, 248, 22-31. doi:10.1007/ s004060050014.

McGorry, P.D. et al. (2007). Investing in youth mental health is a best buy. The Medical Journal of Australia, 187(7), 5-7.

Mishara, A.L., \& Schwartz, M.A. (2013). What Does Phenomenology Contribute to the Debate About DSM-5? In J. Paris, \& J. Phillips. (Eds.). Making the DSM-5 - Concepts and Controversies (pp. 129-142). New York: Springer.

Oliva, M.P. (2014). Transformamos problemas cotidianos em transtornos mentais. Recuperado em 


\section{OBSERVANDO A PSIQUIATRIA}

18 out. 2014 de: < http://brasil.elpais.com/brasil/2014/09/26/sociedad/1411730295_336861. html>.

Oliveira, S.M. (2012). Os alcances e limites da medicalização do risco para a psicose: a emergência de uma nova categoria? Physis Revista de Saúde Coletiva, 22(1), 291-309.

Phillips, J. (2013). Conceptual issues in the classification of psychosis. Current opinion in psychiatry, 26(2), 214-218. doi:10.1097/YCO.0b013e32835d9f0f.

Regier, D.A. et al. (2013, Jan.). DSM-5 field trials in the United States and Canada, part II: Test-retest reliability of selected categorical diagnoses. American Journal of Psychiatry, 170, 59-70. doi:10.1176/appi.ajp.2012.12070999.

Sadler, J.Z. (2005). Values and psychiatric diagnosis. New York: Oxford University Press.

Silva, L.R.F. (2013). Da prevenção ao manejo do risco: contraste e indefinição na história recente da psiquiatria. Rio de Janeiro: Universidade do Estado do Rio de Janeiro.

Woodbridge-Dodd, K., \& Fulford, K.W.M.B. (2004). Whose Values?. London: The Sainsbury Centre for Mental Health.

Yung, A. R. et al. (1996). Monitoring and care of young people at incipient risk of psychosis. Schizophrenia bulletin, 22(2) 283-303, doi:10.1093/schbul/22.2.283.

Yung, A.R. et al. (2012). Whither the attenuated psychosis syndrome? Schizophrenia Bulletin, 38(6), 1130-1134. doi:10.1093/schbul/sbs 108.

Yung, A.R., \& McGorry, P.D. (1996). The Prodromal Phase of First-Episode Psychosis: Past and Current Conceptualizations. Schizophrenia Bulletin, 22(2), 353-370.

Zorzanelli, R.T. et al. (2014). Um panorama sobre as variações em torno do conceito de medicalização entre 1950-2010. Ciência \& Saúde Coletiva, 19(6), 1859-1868. doi:10.1590/141381232014196.03612013 .

\section{Resumos}

(Conflicting values in the production of DSM-5: the "case" of the attenuated psychotic syndrome)

The discussion on the possible inclusion of Attenuated Psychosis Syndrome as an official diagnosis of the DSM-5 was permeated by different values, which underpinned the division between those who argued in favor and those who were against the creation of that new category. The prominence given to certain values generated divergent positions, but which were equally legitimate and well grounded, and which brought up significant questions regarding the potentially innovative scenario of contemporary preventive psychiatry. The ethical preoccupation with suffering, the risks associated with preventive actions and the importance of the role of values in the formulation of diagnostic categories and in psychiatric practice were some of the key issues raised by that debate.

Keywords: Attenuated psychosis syndrome, values, DSM-5, at-risk mental state 
(Conflit de valeurs dans la production de DSM-5: le "cas" du syndrome psychotique atténué)

La discussion sur la possible inclusion du Syndrome Psychotique Atténué comme diagnostique officiel dans le DSM-5 a été traversée par diverses valeurs, autour desquelles les argumentateurs, en faveur ou à l'opposé de la création de cette catégorie, se sont séparés. Les soutenances de telles valeurs ont suscité des positions divergentes, mais aussi légitimes et bien fondées, qui ont conduit à des questionnements importants pour le cadre potentiellement novateur de la psychiatrie préventive contemporaine. Parmi ces valeurs, il existe la préoccupation éthique par rapport à la souffrance, les risques associés aux actions de prévention, l'importance du rôle des valeurs dans la formulation des catégories diagnostiques, ainsi que dans la pratique psychiatrique.

Mots clés: Syndrome psychotique atténué, valeurs, DSM-5, état mental de risque

(Valores en conflicto en la producción de DSM-5: el "caso" del síndrome de la psicosis atenuada)

El debate sobre la posible inclusión del síndrome de psicosis atenuada como un diagnóstico oficial en el DSM-5 fue atravesado por numerosos valores, que dividieron a los que estaban a favor o en contra de la creación de esta nueva categoría. Las defensas de tales valores generaron posturas divergentes, pero igualmente legítimas y con fundamento, y trajeron consigo puntos de discusión importantes para el escenario potencialmente innovador de la psiquiatría preventiva contemporánea, incluyendo la preocupación ética por el sufrimiento, los riesgos asociados a la prevención y la importancia del papel de los valores en la formulación de las categorías diagnósticas y en la propia práctica psiquiátrica.

Palabras claves: Síndrome de la psicosis atenuada, valores, DSM-5, estado mental de riesgo

(Widersprüchliche Werte in der DSM-5 Produktion: der „Fall“ des milden psychotischen Syndrom)

Die Debatte über die mögliche Aufnahme des Milden Psychotischen Syndroms als offizielle Diagnose ins DSM-5 wurde von unterschiedlichen Werten begleitet, die die Meinungen für oder gegen die Schaffung dieser neuen Kategorie spalteten. Die Verteidigung dieser Werte resultierte in gegensätzlichen Stellungen, alle legitim und begründet, die von wichtigen Fragen begleitet waren bezüglich des potentiell innovativen Szenarios der zeitgenössischen vorbeugenden Psychiatrie. Diese Fragen beinhalteten, zum Beispiel, ethischen Bedenken in Bezug auf das Leiden, auf die Risiken im Zusammenhang mit präventiven Maßnahmen und die Bedeutung der Rolle der Werte bei der Formulierung von Diagnosekategorien und in der psychiatrischen Praxis.

Schlüsselwörter: Mildes psychotisches Syndrom, Werte, DSM-5, mentaler Risikozustand 


\section{OBSERVANDO A PSIQUIATRIA}

Citação/Citation: Gonçalves, A.M.N., Dantas, C. de R., \& Banzato, C.E.M. (2015, março). Valores conflitantes na produção do DSM-5: o "caso" da síndrome psicótica atenuada. Revista Latinoamericana de Psicopatologia Fundamental, 18(1), 139-151.

Editor do artigo/Editor: Prof. Dr. Cláudio E.M. Banzato e Profa. Dra. Rafaela Zorzanelli

Recebido/Received: 15.01.2015/ 01.15.2015 Aceito/Accepted: 5.2.2015 / 2.5.2015

Copyright: (C) 2009 Associação Universitária de Pesquisa em Psicopatologia Fundamental/ University Association for Research in Fundamental Psychopathology. Este é um artigo de livre acesso, que permite uso irrestrito, distribuição e reprodução em qualquer meio, desde que o autor e a fonte sejam citados / This is an open-access article, which permits unrestricted use, distribution, and reproduction in any medium, provided the original authors and sources are credited.

Financiamento/Funding: Os autores declaram não terem sido financiados ou apoiados / The authors have no support or funding to report.

Conflito de interesses/Conflict of interest: Os autores declaram que não há conflito de interesses / The authors have no conflict of interest to declare.

\section{Arthur Maciel Nunes Gonçalves}

Psiquiatra; Mestrando do Departamento de Psicologia Médica e Psiquiatria da Faculdade de Ciências Médicas da Universidade Estadual de Campinas - Unicamp (Campinas, SP, Br).

Rua Tessália Vieira de Camargo, 126 - Cidade Universitária “Zeferino Vaz"

13083-887 Campinas, SP, Br

e-mail: arthurmaciel@gmail.com

\section{Clarissa de Rosalmeida Dantas}

Psiquiatra; Doutora em Ciências Médicas, Professora do Departamento de Psicologia Médica e Psiquiatria da Faculdade de Ciências Médicas da Universidade Estadual de Campinas Unicamp (Campinas, SP, Br).

Rua Tessália Vieira de Camargo, 126 - Cidade Universitária “Zeferino Vaz"

13083-887 Campinas, SP, Br

e-mail: crdantas@fcm.unicamp.br

\section{Cláudio E.M. Banzato}

Psiquiatra; Doutor em Filosofia; Professor Associado do Departamento de Psicologia Médica e Psiquiatria da Faculdade de Ciências Médicas da Universidade Estadual de Campinas Unicamp (Campinas, SP, Br).

Rua Tessália Vieira de Camargo, 126 - Cidade Universitária “Zeferino Vaz"

13083-887 Campinas, SP, Br

e-mail: cbanzato@fcm.unicamp.br 